\title{
Evidencia de validez e invarianza del Mini - International Personality Item Pool (Mini-IPIP)
}

\author{
Validity evidence and invariance of the Mini - International Personality \\ Item Pool (Mini-IPIP) \\ Daniel Edgardo Yupanqui-Lorenzo ${ }^{11}$, Víctor Pulido Capurro², \\ Edith Olivera Carhuaz ${ }^{3}$ \\ Universidad César Vallejo, Lima, Perú ${ }^{1}$ \\ Universidad Privada San Juan Bautista, Lima, Perú \\ Universidad Le Cordon Bleu, Lima, Perú ${ }^{3}$ \\ Orcid ID: https://orcid.org/0000-0002-8977-2888 ${ }^{1}$ \\ Orcid ID: https://orcid.org/0000-0002-9238-5387² \\ Orcid ID: https://orcid.org/0000-0002-7400-8625
}

Recibido: 21 de mayo de 2021

Aceptado: 11 de agosto de 2021

\begin{abstract}
Resumen
Existe una necesidad de instrumentos breves sobre la personalidad en el contexto peruano. Por lo cual, el objetivo del estudio es analizar la validez del Mini - International Personality Item Pool (Mini-IPIP) y su invarianza factorial. El método utilizado fue instrumental. Los participantes fueron 521 estudiantes de una universidad de Lima. El instrumento utilizado fue el Mini-IPIP, compuesto por 20 ítems con cinco opciones de respuesta. Se utilizó el análisis factorial confirmatorio e invarianza factorial a través del software $\mathrm{R}$ studio. Los resultados obtenidos mostraron que el modelo final (MF) obtuvo mejor índices de ajuste que los otros modelos probados (MP, M1 y M2). La extracción de ítems en el MF incrementó la fiabilidad de los factores. La invarianza factorial determinó que no existen diferencia entre el género. En conclusión, el MF se ajusta mejor a la realidad de la muestra peruana al obtener mayores valores de los índices de bondad de ajuste.
\end{abstract}

Palabras clave: Big-Five; personalidad; Mini-IPIP; IPIP-FFM; validez.

\section{Abstract}

There is a need for brief instruments on personality in the Peruvian context. Therefore, the objective of the study is to analyze the validity of the Mini - International Personality Item Pool (Mini-IPIP) and its factorial invariance. The method used was instrumental. The participants were 521 university students from a university in Lima. The instrument used was the MiniIPIP, consisting of 20 items with five response options. Confirmatory factor analysis and factorial invariance was used through R studio software. The results showed that the final model

${ }^{1}$ Correspondencia al autor:

dyupanquil@ucvvirtual.edu.pe 
(MF) obtained better fit rates than the other tested models (MP, M1 and M2). The extraction of items in the MF increased the reliability of the factors. Factorial invariance determined that there is no difference between gender. In conclusion, the MF is better suited to the reality of the Peruvian sample by obtaining higher values from goodness-of-fit indices.

Keywords: Big-Five; personality; Mini-IPIP; IPIP-FFM; validity.

\section{Introducción}

La personalidad es un constructo que ha llamado la atención a un gran número de psicológicos investigadores. Han sido diversas las investigaciones que trataron de comprender su origen, sentido conceptual, funcionalidad y medición. A lo largo del tiempo se plantearon diversos modelos teóricos entre los cuales algunos mantienen su vigencia científica (e.g. Cattell, 1943; Fiske, 1949; Goldberg, 1981; McCrae \& Costa, 1987; Norman, 1963). Entre las propuestas teóricas, la de mayor uso dentro de la investigación científica ha sido la teoría del Big-Five (Gosling et al., 2003). Esta teoría propone cinco tipos de personalidad: extraversión, neuroticismo, apertura a la experiencia, agradabilidad y responsabilidad. Desde su concepción, algunos autores como John et al. (1991), Costa \& McCrae (1992) y Goldberg (1992) han desarrollado instrumentos de medición a partir de esos cinco componentes. Aunque han existido otros instrumentos que evalúan mayor número de personalidades (e.g. 16 PF, MMPI, etc.), los que se elaboraron en base al Big-Five han tenido mayor acogida por su factibilidad en el proceso científico.

Por su parte, los instrumentos que miden rasgos, tipos, o trastornos de personalidad se caracterizan por poseer una gran cantidad de ítems; esto se debe a la complejidad de los constructos. Como consecuencia del número extenso de preguntas y el tiempo que toma responderlas, el evaluado siente cansancio, aburrimiento, irritación y desmotivación (Wise \& DeMars, 2005), razón por la cual podría provocar errores en la medición. En este caso, algunos de los instrumentos con numerosos ítems son: el inventario Big-Five, Inventario Multiaxial de Millón, MMPI-2, Inventario de Eysenck, NEO-PI (y sus versiones revisadas), CPI, 16PF, entre otros. Para ser precisos, el instrumento de Costa \& McCrae (1992) el NEO-PI está constituido por 240 ítems que miden cinco dimensiones de personalidad basados en el Big-Five, con un tiempo aproximado de 60 minutos para completarlo. En su versión revisada (NEO-PI-R) disminuye el número de ítems, y su versión abreviada (NEO-FFI) comprende 60 ítems, los cuales siguen manteniendo un alto número de indicadores. Otro instrumento extenso es el inventario clínico multiaxial de Millon (1997) con 175 ítems; el de Eysenck \& Eysenck (1964) con 60 ítems; el de Goldberg (1992) con 100 ítems; y, el Big-Five Inventory (BFI) de 44 ítems 
(John et al., 1991). Cabe mencionar que existen otros instrumentos sobre personalidad que, en su mayoría, presentan copyright de sus ítems lo cual no permite su uso sin permiso de autoría (p.e. MMPI, CPI, 16PF y NEO-PI) (Goldberg, 1999), de esa forma, se restringe su libre estudio en la ciencia.

Anteriormente se consideraba que los instrumentos compuestos por numerosos ítems mostraban mejores propiedades psicométricas en comparación de los instrumentos cortos o breves. No obstante, esta superioridad estadística no se demostraba al emplearse en investigaciones (Burisch, 1997). En contraparte, se evidenció la viabilidad y factibilidad de los instrumentos breves utilizados para la investigación científica y social (Goldberg, 1999). Además, los instrumentos breves evitan la redundancia de indicadores, lo cual permite reducir la fatiga, aburrimiento y frustración a los evaluados (Robins, Hendin, et al., 2001). En este caso, los psicometristas han probado que las escalas cortas muestran mayor o igual evidencia de validez psicométrica (Campbell \& Fiske, 1959; Robins, Tracy, et al., 2001; Russell et al., 1989); de ese modo se refuta la suposición de que los instrumentos largos sean superiores.

\section{Aplicabilidad del International Personality Item Pool}

La presente investigación consideró la propuesta de Goldberg (1999) denominado International Personality Item Pool - Five Factor Model (IPIP-FFM), el cual se compone por 50 ítems. Este instrumento fue abreviado por Donnellan et al. (2006), quienes propusieron una versión de 20 ítems denominado Mini-IPIP. Estos autores realizaron un estudio donde mostraban las propiedades métricas de los instrumentos junto con el TIPI (Ten-Item Personality Inventory) el cual es un instrumento de 10 ítems. Se obtuvo que el IPIP-FFM y el Mini-IPIP mostraron similares medidas en su distribución y cargas factoriales. Además, el Mini-IPIP probó poseer un adecuado índice de bondad de ajuste en su composición de cinco factores y cuatro indicadores para cada factor. La evidencia de validez basado en el criterio para variables como la ansiedad, depresión, agresión, afecto positivo, afecto negativo y satisfacción con la vida mostraron índices de correlación similares entre IPIP-FFM y Mini-IPIP. De esa manera, se concluyó que el Mini-IPIP es una herramienta útil para evaluar los cincos grandes personalidades en procesos de investigación, debido a que sus propiedades psicométricas resultaron ser idénticas al IPIP-FFM.

El Mini-IPIP ha sido utilizado en diversas investigaciones psicométricas en diferentes países. Luego de la publicación de Donnellan (2006), Cooper et al., (2010) realizaron un estudio sobre las propiedades psicométricas de dicho instrumento, lo cual arrojó adecuados índices de ajuste al modelo de cinco factores de personalidad; sin embargo, se evidenció dificultad en la 
confiabilidad del factor responsabilidad. Posteriormente, Sibley et al. (2011) extendió el estudio del Mini-IPIP, en su análisis factorial exploratorio (AFE) obteniendo seis factores con una rotación ortogonal; para su análisis factorial confirmatorio (AFC) propuso la rotación oblicua. En el estudio de comparación, fue el modelo de seis factores correlacionados el que poseía mejores índices de bondad de ajuste. No obstante, fue un adecuado inicio de revisión de las propiedades psicométricas del Mini-IPIP. Por ello, Carretta et al. (2012) deseó estudiar si el inventario puede poseer un factor único. Los resultados fueron sobreestimados y no se llegó a una conclusión adecuada sobre un factor común entre los cinco tipos de personalidades.

Ese mismo año, Li et al. (2012) puso a prueba el modelo de cinco factores correlacionados -composición factorial inicial del Mini-IPIP- y los resultados que obtuvieron demostró adecuados índices de ajuste y la correlaciones entre los factores fueron estables; de igual modo, la consistencia interna medida por el coeficiente omega estuvieron por encima de lo esperado (.70). Igualmente, el estudio de Baldasaro et al. (2013) demostró la existencia de cinco factores, además, expuso evidencia de validez basado en la relación con otras variables como el estrés percibido, hostilidad, abuso de alcohol, dependencia al alcohol y delincuencia. Inclusive, ese mismo año se publicó la primera investigación psicométrica del Mini-IPIP en la versión traducida al francés. Este estudio sustentó la versión de cinco factores, y se realizó invarianza factorial utilizando datos sociodemográficos, los cuales fueron adecuados (Laverdière et al., 2013).

En el 2014, se siguió investigando sobre el Mini-IPIP, y una de esas investigaciones demostró satisfactorios niveles en la calidad de medición de los cinco factores (Jones, 2014); una investigación, fue un estudio de reducción de la versión de 50 ítems a una versión de 20 ítems (Topolewska et al., 2014). Estos estudios expuestos fueron investigaciones de un largo intervalo de tiempo, sobre el inicio de las evaluaciones psicométricas del Mini-IPIP.

Las investigaciones actuales que corresponden al estado del arte sobre los estudios psicométricos del Mini-IPIP inician con el trabajo de Martínez-Molina \& Arias (2018) quienes realizaron una investigación compleja sobre las versiones traducidas al español del Mini-IPIP. Estas dos versiones se compusieron por los mismos ítems, una de versión de ítems positivos y el otro de ítems positivos e inversos. Los resultados mostraron que la versión de ítems positivos (con errores correlacionados) tuvo mejor índices de ajuste que la otra versión (con errores correlacionados). Además, se realizó un estudio de invarianza cross-cultural con una muestra chilena y norteamericana. Luego, el estudio de Leong et al. (2019), sobre el Mini-IPIP adaptado para Malasia, confirmó la composición estructural de cinco factores. El modelo obtuvo 
adecuados índices de ajuste y se obtuvo evidencia de validez basado en la relación con otras variables.

Ese mismo año, Oliveira (2019) adaptó el Mini-IPIP al portugués y brindó adecuados índices de ajuste del modelo de cinco factores, aunque tuvo un problema similar al de Cooper et al., (2010) con la fiabilidad del factor responsabilidad. Finalmente, en el 2020 se publicaron cinco investigaciones sobre el Mini-IPIP. La primera fue una versión adaptada a una muestra polaca, lo cual evidenció ajustes adecuados del modelo (Czerwiński \& Atroszko, 2020). La segunda versión del instrumento fue adaptada al francés, en el cual reafirmaron que la versión francesa del 2013, mantuvo su estructura factorial con adecuados índices de ajuste y fiabilidad por consistencia interna (Laverdière et al., 2020). El tercer estudio comparó el Mini-IPIP con BFI Facet Scales, por lo que el Mini-IPIP se elaboró en cuatro factores, y en el análisis de invarianza obtuvo menores valores en sus índices de ajuste a comparación del BFI Facet Scales (Ock et al., 2020). El cuarto estudio estuvo dirigido a una población con cáncer, en el cual se probaron dos modelos: uno de cinco factores y otro de cinco factores con errores correlacionados. El modelo reespecificado fue el que obtuvo mejor índices de ajuste y fiabilidad (Perry et al., 2020). Por último, el quinto estudio fue desarrollado en una población de argentinos por Simkin et al. (2020), lo cual obtuvo índices de confiabilidad superiores a .70. Sus índices de bondad de ajuste fueron satisfactorios; y dentro de su invarianza factorial llegó a una invarianza fuerte. De esa manera, a nivel internacional, los estudios del Mini-IPIP han mostrado tener relevancia dentro de sus propiedades métricas.

\section{Instrumentos sobre personalidad en el Perú}

En el contexto nacional, los instrumentos utilizados en investigaciones sobre la personalidad han sido el NEO-PI-R (Cassaretto, 2011; Rosas et al., 2001), el Inventario de personalidad de Millon (MIPS) (Gastelumendi \& Oré, 2013), Cuestionario de personalidad de Eysenck para niños (EPQ-J)(Hernández Muro, 2013), EPI-Forma A, Cuestionario de Personalidad Situacional (Araujo, 2014), Scanning Neuropsicológico de la Personalidad (Sarria-Joya et al., 2011; Vizcarra Fernández et al., 2015), entre otros. Cabe precisar que estos instrumentos mencionados poseen gran cantidad de ítems, por lo cual Anicama \& Chumbimuni (2018) prestaron interés en la teoría del Big-Five, desarrollando el Cuestionario de la Personalidad de los Cinco Grandes (CBP-LRC). Sin embargo, entre todos los instrumentos utilizados que han mostrado evidencia psicométrica en el Perú, fue Dominguez-Lara et al. (2018) quienes estudiaron el Big-Five en su versión breve de 37 ítems. Aunque dicha versión fue publicada como un estudio preliminar, ese mismo año Dominguez-Lara \& Merino-Soto (2018) publicaron 
su estudio sobre las propiedades psicométricas de los inventarios de personalidad del Big-Five en versión de 15 ítems y 10 ítems, los cuales mostraron adecuadas propiedades métricas en las dos versiones. Los índices de confiabilidad fueron óptimos en ambos instrumentos y las cargas factoriales mostraron estar por encima de lo esperado ( $>.40)$. De igual manera, estas dos versiones mostraron altos índices del coeficiente de congruencia $(\Phi>.80)$.

Si bien, estos últimos estudios han utilizado instrumentos desarrollados bajo la perspectiva teórica del Big-Five; no obstante, se debe considerar que los instrumentos breves sintetizan información teórica en indicadores específicos, los cuales muestran pocos rasgos de la personalidad. Por ello, explorar un constructo teórico como la personalidad y sus tipos no basta con un instrumento. Por lo tanto, esta investigación observó que en el contexto peruano carece de instrumentos de medición breves sobre la personalidad para utilizarlo en el campo de la ciencia, más que en el campo clínico. Las tantas investigaciones sobre la personalidad en el Perú carecen de instrumentos breves para obtener información con evidencia de validez y asegurar la calidad de los resultados. Inclusive, algunos de estos instrumentos breves descritos en el párrafo anterior han mostrado tener inconsistencia en su confiabilidad. Por ello, al ser utilizados los investigadores se arriesgan a medir con altas probabilidad de error. No obstante, el Mini-IPIP ha mostrado estabilidad dentro de sus coeficientes de confiabilidad y su composición factorial, en las investigaciones internacionales.

A la luz de estos antecedentes, se ha vuelto pertinente estudiar el Mini-IPIP en el Perú, debido a los carentes inventarios de personalidad y las carencias en sus propiedades psicométricas. Es por ello que la presente investigación tiene el objetivo de analizar la evidencia de validez basada en la estructura interna del Mini-IPIP; a su vez, se pretende realizar un análisis de invarianza factorial. En este caso, se utilizó el Mini-IPIP en su versión traducida al español con ítems positivos. A diferencia de la versión con ítems inversos, este ha demostrado mayores índices de ajuste y de invarianza factorial en una muestra chilena (Martínez-Molina \& Arias, 2018).

\section{Metodología}

\section{Diseño}

El presente estudio tiene un diseño instrumental debido al objetivo psicométrico y procesos metodológicos (Ato et al., 2013).

\section{Participantes}

La población de estudio fue conformada por 595 estudiantes de una universidad privada de Lima. Para obtener el número de exacto de la muestra, se solicitó a la secretaría académica el 
número de matriculados en el periodo 2021-1. Luego de obtener la cantidad exacta de estudiantes inscritos, se procedió a calcular el tamaño muestral. Para ello, se consideró un 99\% de nivel de confianza y un margen de error del 2\%. El muestreo fue probabilístico aleatorio simple (Sánchez \& Reyes, 2015). Como resultado se obtuvo a 521 estudiantes universitarios limeños. Como se observa en la tabla 1 , la muestra se compuso por $41.7 \%$ de varones y $58.3 \%$ de mujeres.

Las edades fluctuaron entre los 18 a 35 años siendo la media de edad los 22.62 años (DE $=4.74)$. Los rangos de edades muestran que un $63.5 \%$ son adolescentes entre 18 a 23 años; el 28.2\% son adultos jóvenes de entre 24 a 29 años; y, el 8.3\% son adultos medios entre 30 a 35 años. La situación laboral de los estudiantes muestra que el $52.4 \%$ solo se dedica al estudio mientras que el $47.6 \%$ estudia y trabaja. El estado civil de los evaluados muestra que el 76.6\% son solteros, 20.0\% están en una relación de pareja, 3.1\% son casados y $0.4 \%$ son divorciados. Se evaluaron estudiantes de matrícula regular e irregular, y del turno diurno (57.2\%) y nocturno (42.8\%).

\section{Tabla 1}

Datos sociodemográficos de la muestra

\begin{tabular}{llcc}
\hline \multicolumn{1}{c}{ Categoría } & \multicolumn{1}{c}{ Grupos } & $f$ & $\%$ \\
\hline Sexo & Hombre & 217 & $41.7 \%$ \\
& Mujer & 304 & $58.3 \%$ \\
Edad $^{\text {a }}$ & 18-23 años & 331 & $63.5 \%$ \\
& 24-29 años & 147 & $28.2 \%$ \\
& 30-35 años & 43 & $8.3 \%$ \\
Eaboral & Solo estudia & 273 & $52.4 \%$ \\
& Estudia y trabaja & 248 & $47.6 \%$ \\
& Soltero & 399 & $76.6 \%$ \\
& En relación & 104 & $20.0 \%$ \\
& Casado & 16 & $3.1 \%$ \\
& Divorciado & 2 & $0.4 \%$ \\
& Diurno & 298 & $57.2 \%$ \\
& Nocturno & 223 & $42.8 \%$ \\
\hline
\end{tabular}

Nota: ${ }^{a} \bar{x}=22.62 ; \sigma=4.74 ; f=$ frecuencia; $\%=$ porcentaje. 


\section{Instrumento}

El Mini-International Personality Item Pool (Mini-IPIP) es un instrumento que mide cinco tipos de personalidad teóricamente establecidas por el Modelo de los Cinco Grandes (Donnellan et al., 2006). Se trata de una versión reducida del International Personality Item Pool-Five Factor Model (IPIP-FFM) de Goldberg (1992) el cual estuvo compuesta por 50 ítems. La versión Mini-IPIP se conforma por un total de 20 ítems, los cuales se agrupan en cinco factores: extraversión, agradabilidad, responsabilidad, neuroticismo y apertura (intelecto/imaginación). Cada reactivo se compone por una escala de respuesta de cinco puntos (1= Totalmente en desacuerdo; a, 5 = Totalmente de acuerdo).

El Mini-IPIP cuenta con una versión adaptada en países de habla hispana, lo cual permite la adecuación a nivel internacional y no muestra verse influenciado por lenguajes locales que solo sean comprensibles en ciertos países. Esta adaptación posee dos versiones: la primera considera los ítems negativos y el factor neuroticismo; y la segunda, posee ítems positivos incluyendo los de neuroticismo, al cual se le transforma por su opuesto teórico (estabilidad emocional). Martínez-Molina \& Arias (2018) realizaron una investigación de estas dos versiones incluyendo la versión original (inglés). Los resultados mostraron que el instrumento con ítems positivos poseía un mejor índice de bondad de ajuste (RMSEA= .052; CFI= .984; TLI $=.970 ; \chi^{2}=171$ ), a diferencia del instrumento que poseía ítem negativos. Simkin et al. (2020) también utilizó el Mini-IPIP traducida al español y determinó un aceptable ajuste del modelo. Para la presente investigación se tomará en cuenta la versión de ítems positivos.

\section{Procedimiento}

Inicialmente, se envió el proyecto de investigación al comité de ética de la universidad donde se realizó la investigación. Luego de exponer los propósitos de la investigación, la deliberación del comité fue aprobar el proyecto y la aplicación de instrumentos en la muestra de estudio. Para ello se trasladó el instrumento a un formulario en Google. De esa forma se respetó el distanciamiento social y estado de emergencia sanitario en el Perú. Dentro del formulario se estableció un consentimiento informado sobre la participación, junto al detalle de los objetivos de la investigación y sobre el proceso de ética sobre el tratamiento de la información obtenida. No hubo recompensa monetaria a los participantes, solo se consideró la voluntad de los mismo. Se obtuvo el permiso de la institución educativa para poder obtener acceso a los correos y contactos con los estudiantes. El tiempo aproximado de solución del instrumento fue de 10 a 15 minutos. 


\section{Análisis de datos}

En el estudio se utilizó la plataforma de RStudio, donde se ejecutó los paquetes de Lavaan, Psych, SemPlot y SemTools. La exploración de datos se obtuvo mediante el análisis de los resultados descriptivos. Se obtuvo la media, desviación estándar, asimetría y curtosis de los ítems, los cuales mostraron distribución normal con ciertas excepciones. El AFC se ejecutó mediante el estimador de máxima verosimilitud para datos robustos (MLR) con correcciones de la normalidad (Yuan \& Bentler, 1998). Se utilizó dicho estimador por ser viable en variables continuas (escala de tipo Likert de cinco o más opciones de respuesta) (Rhemtulla et al., 2012). Para la evaluación de las cargas factoriales se consideró que estos deberían ser mayor a .40 (Dominguez-Lara, 2018); por lo contrario, serían excluidos del modelo.

Para evaluar los modelos se examinaron los índices de ajuste: chi cuadrado $\left(\chi^{2}\right)$, grados de libertad (gl), índice de ajuste comparativo (CFI), índice Tucker Lewis (TLI), residuo estandarizado cuadrático medio (SRMR) y error cuadrático medio de aproximación (RMSEA) (Browne \& Cudeck, 1992). Para la fiabilidad se utilizaron los coeficientes alfa, omega y H (Choi et al., 2009). Por último, se realizó el análisis de invarianza según el sexo. Para evaluar la invarianza se utilizaron los índices de ajuste: CFI, RMSEA y $\chi^{2}(\mathrm{gl})$. Se consideró un ajuste de CFI igual o mayor a .90 como aceptable y del RMSEA menor a .08. De igual modo se evaluó los cambios dentro de los modelos: configuracional, métrica, fuerte y estricta ( $\triangle \mathrm{CFI}, \triangle \mathrm{RMSEA}$, $\left.\Delta \chi^{2}[\mathrm{gl}]\right)$ (Cheung \& Rensvold, 2002).

\section{Resultados}

\section{Análisis preliminar de ítems}

La tabla 2 evidencia el análisis descriptivo de los ítems. Las medidas de tendencia central muestran que el ítem 20 “Tengo buena imaginación” tiene el valor más alto; por lo contrario, el ítem 16 “Me gusta ser el centro de atención” posee el valor más bajo. Por otra parte, fueron diecisiete los ítems que presentaron desviación estándar mayores a uno. Gran parte de los ítems poseen asimetría negativa, evidenciando una tendencia a puntuaciones altas. En cuanto a la curtosis se mostró que ningún ítem supera la unidad. En consecuencia, en cuanto a la asimetría y la curtosis de los ítems del Mini-IPIP, se consideran de aceptable distribución al encontrarse en el rango de \pm 1.5 ; de esa manera se determina la viabilidad del análisis factorial (George \& Mallery, 2020). 


\section{Tabla 2}

Análisis preliminar de los ítems Mini-IPIP

\begin{tabular}{|c|c|c|c|c|}
\hline Ítems & M & $\sigma$ & g1 & g2 \\
\hline \multicolumn{5}{|l|}{ Extraversión (E) } \\
\hline 1. Soy el alma de la fiesta & 3.11 & 1.09 & -0.08 & -0.22 \\
\hline 6. Hablo mucho & 3.50 & 1.07 & -0.16 & -0.48 \\
\hline 11. En las fiestas hablo con muchas personas & 3.24 & 1.20 & -0.13 & -0.75 \\
\hline 16. Me gusta ser el centro de atención & 2.30 & 1.16 & 0.46 & -0.64 \\
\hline \multicolumn{5}{|l|}{ Agradabilidad (A) } \\
\hline 2. Soy sensible hacia las emociones de otros & 3.48 & 1.10 & -0.33 & -0.52 \\
\hline $\begin{array}{l}\text { 7. Me intereso por los problemas de otras } \\
\text { personas }\end{array}$ & 3.51 & 1.09 & -0.41 & -0.37 \\
\hline 12. Siento las emociones de los otros & 3.33 & 1.10 & -0.20 & -0.57 \\
\hline 17. En realidad me intereso por los demás & 3.61 & 1.02 & -0.52 & -0.01 \\
\hline \multicolumn{5}{|l|}{ Responsabilidad (C) } \\
\hline 3. Realizo mis tareas inmediatamente & 3.19 & 0.99 & 0.11 & -0.22 \\
\hline 8. Suelo poner las cosas en su lugar & 3.72 & 1.02 & -0.42 & -0.36 \\
\hline 13. Me gusta el orden & 3.83 & 1.03 & -0.52 & -0.35 \\
\hline 18. Soy ordenado & 3.62 & 1.14 & -0.44 & -0.54 \\
\hline \multicolumn{5}{|l|}{ Neuroticismo $(N)^{1}$} \\
\hline 4. Tengo pocos cambios de ánimo & 3.09 & 1.19 & -0.24 & -0.76 \\
\hline 9. Estoy relajado la mayor parte del tiempo & 3.13 & 1.26 & -0.15 & -0.97 \\
\hline 14. Difícilmente me molesto & 3.14 & 1.34 & -0.04 & -1.15 \\
\hline 19. Rara vez me siento triste & 3.04 & 1.29 & 0.12 & -1.08 \\
\hline \multicolumn{5}{|l|}{ Apertura $(O)$} \\
\hline 5. Tengo mucha imaginación & 4.05 & 1.00 & -0.87 & 0.20 \\
\hline 10. Me interesan las ideas abstractas & 3.33 & 1.03 & -0.03 & -0.22 \\
\hline 15. Entiendo con facilidad las ideas abstractas & 3.38 & 0.94 & -0.09 & 0.06 \\
\hline 20. Tengo buena imaginación & 4.06 & 0.95 & -0.81 & -0.15 \\
\hline
\end{tabular}

Nota. M: media; $\sigma$ : desviación estándar; g1: asimetría; g2: curtosis.

${ }^{1}$ Versión positiva de $\mathrm{N}$ (Estabilidad emocional)

\section{Análisis factorial confirmatorio}

Cuatro modelos fueron estimados en el estudio: modelo principal (MP), basado en Donnellan et al. (2006), el cual plantea originalmente cuatro ítems para cada factor de personalidad que están correlacionados; modelo 1 (M1) basado en Martínez-Molina \& Arias (2018) quienes argumentan que el modelo español con residuos correlacionados (ítems 2 y 12; ítems 10 y 15) posee mejor ajuste del modelo con ítems negativos; modelo 2 (M2) basado en la reespecificaciones de Perry et al. (2020) quienes añaden correlación entre residuos (ítems 2 y 
12; 7 y 17; 10 y 15; 5 y 20); y, el modelo final (MF) establecido previo análisis del MP (figura 1).

A)

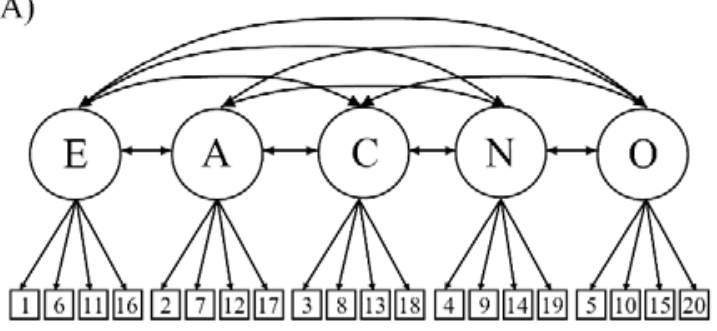

Modelo Principal

(Donnellan et al., 2006)

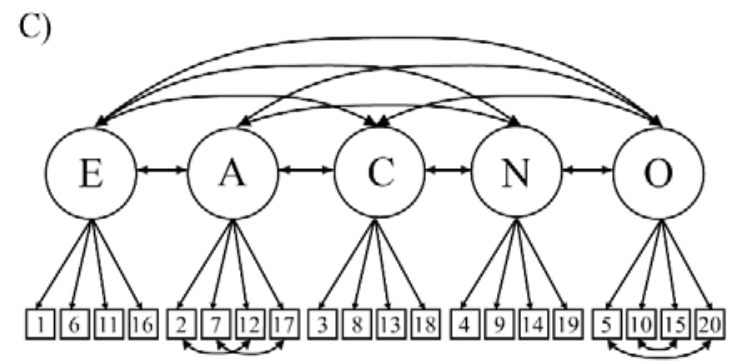

Modelo 2

(Perry et al., 2020)
B)

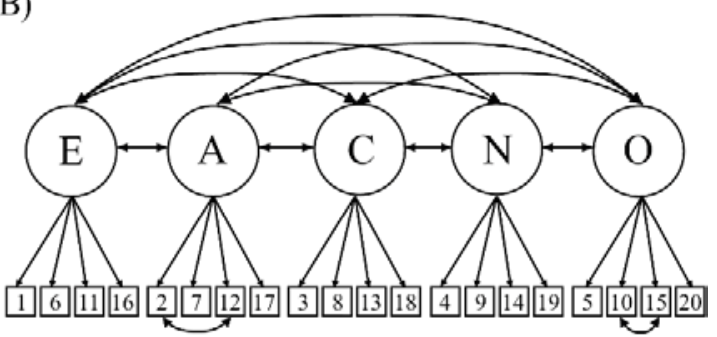

Modelo 1

(Martinez-Molina \& Arias, 2018)

D)

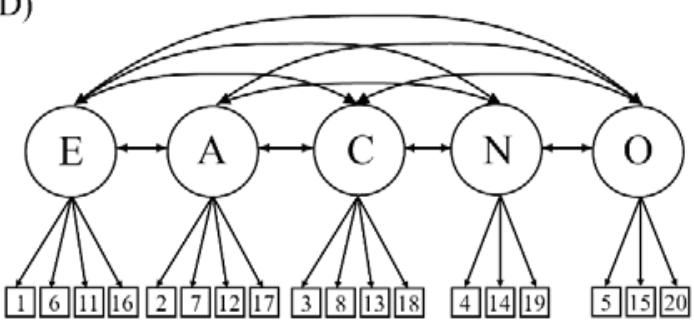

Modelo Final

Figura 1. Modelos factoriales evaluados del Mini-IPIP

Inicialmente, se analizaron los modelos teóricos que se encontraron en la literatura (MP, M1 y M2). Al observar la tabla 3, el MP obtuvo pobre ajuste del modelo ( $\chi^{2}[\mathrm{gl}]=475.3[160], p$ $<.01 ; \mathrm{CFI}=.898 ; \mathrm{TLI}=.879 ; \mathrm{SRMR}=.059 ; \mathrm{RMSEA}=.059$ [IC90\% $.051-.066])$. Era de esperar que el modelo de Donnellan et al. (2006) muestre bajo ajuste del modelo, debido a investigaciones recientes que tuvieron que realizar ajustes del modelo para un mejor desempeño. Por otro lado, el modelo M1 el cual correlaciona los errores de cuatro ítems (ver figura 1), muestra un ajuste aceptable $\left(\chi^{2}[\mathrm{gl}]=396.4[158], p<.01\right.$; CFI $=.925$; TLI = .910; SRMR = .057; RMSEA = .051 [IC90\% .043 - .058]). Del mismo modo, el M2 con residuos correlacionados de ocho ítems, demostró un ajuste adecuado del modelo $\left(\chi^{2}[\mathrm{gl}]=379.6[156], p\right.$ $<.01 ; \mathrm{CFI}=.930 ; \mathrm{TLI}=.915 ; \mathrm{SRMR}=.053 ; \mathrm{RMSEA}=.049$ [IC90\% .042 - .057]).

Este análisis de los modelos permitió determinar un nuevo modelo: modelo final (MF). En todos los modelos se observó que los ítems 9 y 10 no mostraban ser consistentes debido a que poseían cargas menores a .40. De esa forma, se decidió extraer dichos reactivos del MF (ver figura 1). Además, se prefirió no correlacionar errores dado que afecta la fiabilidad de los 
factores. El MF obtuvo adecuados índices de ajuste $\left(\chi^{2}[\mathrm{gl}]=320.9[125], p<.01\right.$; CFI = .934; TLI = .919; SRMR = .054; RMSEA = .051 [IC90\% .043 - .060]) mejores que el M1 y M2. Además, al comparar los AIC de los modelos, es el MF el que presente un valor menor, lo que indicaría que es el modelo con menores valores perdidos.

Tabla 3

Índices de bondad de ajuste de los modelos MP, M1, M2 y MF

\begin{tabular}{|c|c|c|c|c|c|c|c|}
\hline Modelos & $\chi^{2}$ & $\mathrm{gl}$ & CFI & TLI & SRMR & $\begin{array}{l}\text { RMSEA } \\
\text { [IC 90\%] }\end{array}$ & AIC \\
\hline$M P$ & $475.3^{* *}$ & 160 & .898 & .879 & .061 & $\begin{array}{c}.059 \\
{[.051-} \\
.066]\end{array}$ & 29089.8 \\
\hline M1 & $396.4^{* *}$ & 158 & .925 & .910 & .057 & $\begin{array}{c}.051 \\
{[.043-} \\
.058]\end{array}$ & 29014.9 \\
\hline M2 & $379.6^{* *}$ & 156 & .930 & .915 & .053 & $\begin{array}{c}.049 \\
{[.042-} \\
.057]\end{array}$ & 29002.1 \\
\hline$M F$ & $320.9^{* *}$ & 125 & .934 & .919 & .054 & $\begin{array}{c}.051 \\
{[.043-} \\
.060]\end{array}$ & 25946.9 \\
\hline
\end{tabular}

Nota. $\chi^{2}$ : chi cuadrado; gl: grado de libertad; CFI = índice de ajuste comparativo; TLI = índice Tucker Lewis; SRMR = residuo estandarizado cuadrático medio; RMSEA = error cuadrático medio de aproximación; AIC: criterio de comparación de Akaike. ${ }^{* *} p<.001$

El análisis de los índices de ajuste, permitieron comprender que los modelos con errores correlacionados encontrados en la literatura (M1 y M2) son aceptables. De esa forma, en la tabla 4 se presenta las cargas factoriales de los modelos que han poseído ajustes aceptables. En el M1, M2 y MF las cargas factoriales de los ítems en E, A y O fueron adecuadas $(\lambda>.40)$. Los factores $\mathrm{N}$ y $\mathrm{O}$, de los modelos M1 y M2, mostraron cargas débiles $(\lambda<.40)$ en los ítems 9 y 10. De esa forma, para el MF se extrajeron dichos ítems.

Por un lado, el análisis de la confiabilidad del factor E fueron aceptables en todos los modelos $(\alpha=.73 ; \omega=.73 ; H=.76)$. En el factor $\mathrm{A}$, se ha mostrado cierta variación de un modelo al otro, siendo el MF el que posee mejores índices de confiabilidad $(\alpha=.76 ; \omega=.76$; $H=$.77). De la misma forma pasa con el factor $\mathrm{C}$, donde se observa que el MF obtiene una mejor confiabilidad ( $\alpha=.81 ; \omega=.82 ; H=.88$ ). Cabe precisar, que los coeficientes del M1 y M2 para con los factores A, E y C han sido dentro de lo esperado (>.70). Por otro lado, la fiabilidad del factor $\mathrm{N}$ en todos los modelos se mostró débil; sin embargo, hubo un incremento en los coeficientes para con el MF $(\alpha=.71 ; \omega=.71 ; H=.80)$. Por último, el factor O en el M1 
y M2 presentó índices de confiabilidad débiles (<.70). La extracción del ítem 10, favoreció considerablemente a la fiabilidad obteniendo un coeficiente adecuado $(\omega=.73)$; además, debido a que el ítem 15 poseyó una carga factorial de .395, se vio conveniente utilizar el coeficiente $H$ (Dominguez-Lara, 2016) el cual resultó estar por encima de lo esperado $(H=.83)$ (Tabla 4). 


\section{Tabla 4}

Cargas factoriales y coeficientes de fiabilidad de los modelos M1, M2 y MF

\begin{tabular}{|c|c|c|c|c|c|c|c|c|c|c|c|c|c|c|c|}
\hline \multirow{2}{*}{ Ítems } & \multicolumn{3}{|c|}{$\mathrm{E}$} & \multicolumn{3}{|c|}{ A } & \multicolumn{3}{|c|}{$\mathrm{C}$} & \multicolumn{3}{|c|}{$\mathrm{N}$} & \multicolumn{3}{|c|}{$\mathrm{O}$} \\
\hline & M1 & M2 & MF & M1 & M2 & MF & M1 & M2 & MF & M1 & M2 & MF & M1 & M2 & MF \\
\hline 1 & .642 & .641 & .643 & & & & & & & & & & & & \\
\hline 6 & .654 & .643 & .655 & & & & & & & & & & & & \\
\hline 11 & .762 & .771 & .760 & & & & & & & & & & & & \\
\hline 16 & .477 & .479 & .477 & & & & & & & & & & & & \\
\hline 2 & & & & .529 & .612 & .594 & & & & & & & & & \\
\hline 7 & & & & .755 & .633 & .721 & & & & & & & & & \\
\hline 12 & & & & .627 & .751 & .684 & & & & & & & & & \\
\hline 17 & & & & .686 & .593 & .668 & & & & & & & & & \\
\hline 3 & & & & & & & .499 & .499 & .499 & & & & & & \\
\hline 8 & & & & & & & .650 & .651 & .650 & & & & & & \\
\hline 13 & & & & & & & .817 & .816 & .817 & & & & & & \\
\hline 18 & & & & & & & .895 & .895 & .894 & & & & & & \\
\hline 4 & & & & & & & & & & 639 & 632 & .645 & & & \\
\hline 9 & & & & & & & & & & .333 & .341 & - & & & \\
\hline 14 & & & & & & & & & & .658 & .681 & 395 & & & \\
\hline 19 & & & & & & & & & & 693 & .673 & .869 & & & \\
\hline 5 & & & & & & & & & & & & & .767 & .491 & .769 \\
\hline 10 & & & & & & & & & & & & & .293 & .365 & - \\
\hline 15 & & & & & & & & & & & & & .395 & .528 & .395 \\
\hline 20 & & & & & & & & & & & & & .871 & .590 & .869 \\
\hline$\alpha$ & .73 & .73 & .73 & .76 & .76 & .76 & .81 & .81 & .81 & .66 & .66 & .71 & .69 & .69 & .69 \\
\hline$\omega$ & .73 & .73 & .73 & .72 & .72 & .76 & .82 & .82 & .82 & .68 & .68 & .71 & .63 & .48 & .73 \\
\hline$H$ & .76 & .76 & .76 & .77 & .76 & .77 & .88 & .88 & .88 & .71 & .71 & .80 & .83 & .58 & .83 \\
\hline
\end{tabular}

Nota. E: extraversión; A: agradabilidad; C: responsabilidad; N: neuroticismo (versión positiva); O: Apertura; $\alpha$ : alfa multidimensional; $\omega$ : coeficiente omega; $H$ : coeficiente 


\section{Invarianza factorial de medición}

En la tabla 5, se presenta la invarianza de medición de acuerdo con el género. Se evaluó de forma progresiva la invarianza configuracional $\left(M_{1}\right)$, métrica $\left(M_{2}\right)$, fuerte $\left(M_{3}\right)$ y estricta $\left(M_{4}\right)$. El análisis de invarianza entre hombres y mujeres mostraron índices de ajustes aceptables; en especial, las mujeres obtuvieron mejores ajustes. Se estudio el primer modelo configuracional $\left(M_{1}\right)$, el cual fue óptimo $\left(\chi^{2}[\mathrm{gl}]=425.9[250]\right.$; RMSEA= .056 [.046 - .065]; CFI =.923). El $M_{1}$ sirvió como referencia para aplicar las restricciones. Al restringir las cargas factoriales $\left(M_{2}\right)$ se obtuvo índices de ajuste adecuados $\left(\chi^{2}[\mathrm{gl}]=434.6[263]\right.$; RMSEA $=.054$ [.045 - .063]; CFI $=.920)$ mostrando una mejora en el CFI y RMSEA $(\triangle \mathrm{RMSEA}<.01, \Delta \mathrm{CFI}<.01$; Cheung \& Rensvold, 2002). Se prosiguió con restringir la equivalencia de interceptos ( $\left.M_{3}\right)$, este mostró una disminución en el CFI (.911) y aumento el RMSEA (.057) pero se encontraron dentro de lo esperado ( $\triangle \mathrm{RMSEA}<.01, \Delta \mathrm{CFI}<.01)$. Finalmente, se concluyó con el modelo estricto, el cual demostró mantenerse por debajo de lo esperado en los cambios de los estimadores $(\triangle \mathrm{RMSEA}<.01, \Delta \mathrm{CFI}<.01)$. De esa forma, se determina que el modelo muestra que no existen diferencias entre hombres y mujeres a un nivel estricto.

\section{Tabla 5}

Invarianza factorial del modelo final

\begin{tabular}{lcccccc}
\hline & $\chi^{2}(\mathrm{gl})$ & $\Delta \chi^{2}(\mathrm{gl})$ & RMSEA [IC 90\%] & $\Delta$ RMSEA & CFI & $\Delta$ CFI \\
\hline Hombres & $219.7(125)$ & - & $.062[.048-.076)$ & - & .910 & - \\
Mujeres & $206.6(125)$ & - & $.050[.038-.062]$ & - & .933 & - \\
$M_{1}$ & $425.9(250)$ & - & $.056[.046-.065]$ & - & .923 & - \\
$M_{2}$ & $434.6(263)$ & $8.7(13)$ & $.054[.045-.063]$ & .002 & .920 & .003 \\
$M_{3}$ & $478.4(276)$ & $43.8(13)$ & $.057[.048-.065]$ & -.003 & .911 & .009 \\
$M_{4}$ & $512.0(294)$ & $33.6(18)$ & $.057[.049-.065]$ & .000 & .910 & .001 \\
\hline
\end{tabular}

Nota. $M_{1}$ : configuracional; $M_{2}$ : métrica; $M_{3}$ : fuerte; $M_{4}$ : estricto. 


\section{Discusión}

El objetivo principal del estudio fue evaluar las estructuras factoriales establecidas por la literatura del instrumento Mini-IPIP, el cual ha sido considerado en diversos estudios y traducidos a diferentes lenguas. El análisis de las cargas factoriales del MP, M1 y M2 determinaron que los ítems 9 y 10 mantenían débil saturación a su factor. Lo cual confirma el estudio francés que evaluó el Mini-IPIP, en el cual se obtuvieron cargas débiles de dichos ítems (Laverdière et al., 2020). Por lo tanto, se decidió extraer esos ítems, obteniendo el modelo final (MF).

El modelo principal (MP) establecido por Donnellan et al. (2006) no ha presentado adecuados índices de ajuste, lo que coincide con algunos estudios (Leong et al., 2019; Oliveira, 2019; Perry et al., 2020) y difiere de otros (Cooper et al., 2010; Czerwiński \& Atroszko, 2020; Li et al., 2012; Simkin et al., 2020). Por otro lado, dentro del estado del arte se hallaron dos modelos (M1 y M2) que correlacionaban errores para obtener un buen ajuste del Mini-IPIP (Martínez-Molina \& Arias, 2018; Perry et al., 2020); al calcular estos dos modelos se halló que los errores correlacionados mejoraban la bondad de ajuste, pero afectaban la fiabilidad. En especial el modelo M2, el cual mantenía mayor número de errores correlacionados, mostró disminución en el coeficiente de confiabilidad de apertura.

Debido a dichas dificultades, se evaluó un modelo propio de la investigación: modelo final (MF). Este modelo obtuvo coeficientes de fiabilidad con mejoría en ciertos factores. A diferencia de los modelos M1 y M2, donde se halló valores pobres de fiabilidad en neuroticismo. En el MF, al extraer el ítem 9, el coeficiente alfa y omega superaron el límite aceptable (.71). Sin embargo, otros estudios muestran que el neuroticismo es el factor con menor índices de fiabilidad; por ejemplo, el estudio de Baldasaro et al. (2013), Wielkiewicz (2015) y Martínez-Molina \& Arias (2018) obtuvieron un coeficiente bajo ( $\alpha=.62 ; \alpha=.64 ; \alpha=.65$, respectivamente), seguidamente argumentan que dichos resultados pueden deberse a la complejidad de medir un constructo con pocos ítems. Asimismo, el estudio argentino mostró evidencias aceptables del coeficiente omega en neuroticismo (.77). Por otro lado, lo mismo se puede observar en apertura, para el MF, el coeficiente alfa estuvo por debajo de .70, pero el omega estuvo por encima de lo esperado.

No obstante, estos resultados no se alejan de la literatura, ya que en otros estudios se ha determinado que el neuroticismo y apertura poseen coeficientes que bordean el límite de lo aceptable (.70) (Czerwiński \& Atroszko, 2020; Li et al., 2012). A diferencia de estudios que han mostrado que los factores con menos fiabilidad es la apertura (Laverdière et al., 2020; Topolewska et al., 2014) y responsabilidad (Cooper et al., 2010; Oliveira, 2019; Wielkiewicz, 
2015). Este hecho es común cuando se realizan reducción de ítems, dado que afecta la confiabilidad (Dominguez-Lara \& Merino-Soto, 2018). Un ejemplo claro es el trabajo de Blanken et al. (2018) donde muestra la versión Mini-IPIP y IPIP-NEO-120, y se observa una clara reducción de la confiabilidad entre la versión de 120 ítems y el de 20 ítems.

No obstante, el MF al obtener adecuados índices de ajustes permitió realizar un análisis de invarianza de acuerdo con el género de los participantes. El proceso de invarianza mostró que tanto el grupo de hombres y mujeres presentaron adecuados índices de ajuste, lo que permitió proseguir con el análisis. Al cabo del proceso, se obtuvo la invarianza estricta. Se observó que los cambios en los índices de ajuste estuvieron en lo esperado. De ese modo, se logró determinar la invarianza de medición de la personalidad en dichos grupos; al igual que el estudio argentino (Simkin et al., 2020). Por consiguiente, se obtuvo que el MF fue invariante entre grupos nominales como el sexo. Czerwiński \& Atroszko (2020) mostraron evidencia de invarianza entre grupos de mujeres y hombres, empleados y adolescentes; ambos análisis determinaron índices adecuados y cambios en los índices de ajuste dentro de lo esperado. Por lo tanto, el análisis de invarianza factorial en el estudio permite concluir que no existe diferencias entre los grupos de hombres y mujeres sobre el modelo final.

La última versión del Mini-IPIP, presentó algunas diferencias a nivel de fiabilidad en relación con otras medidas breves sobre personalidad que utilizan la teoría Big-Five como en el caso del BFI-15 y BFI-10. El BFI-15 (3 ítems por factor) presentan bajos coeficientes de fiabilidad en amabilidad, responsabilidad y neuroticismo; lo mismo se identificó en el BFI-10 (2 ítems por factor) los coeficientes omega fueron menores a .70 (Dominguez-Lara \& MerinoSoto, 2018). A comparación de la versión del Mini-IPIP, los otros dos inventarios no son consistentes en su fiabilidad-seguramente por error de medición y reducción de ítems. Por otro lado, el estudio de Dominguez-Lara et al. (2018) en su BFI-37, el cual posee mayor número de ítems en comparación del BFI-15 y BFI-10, los resultados del coeficiente omega son similares a los del Mini-IPIP; no obstante, el BFI-37 tuvo problemas con el omega de amabilidad, mientras que el Mini-IPIP no tuvo ninguna dificultad en relación con la confiabilidad de sus factores. Por ello, se determina que el Mini-IPIP representa de mejor forma y en mayor medida las características y rasgos de personalidad a diferencia de otras medidas breves en el contexto peruano.

En suma, la diferencia entre otros instrumentos y el Mini-IPIP, no solo queda diferenciado en los datos. La interpretación de los rasgos de personalidad del Big-Five que se miden a través del Mini-IPIP permite asegurar que los indicadores de responsabilidad, miden fielmente las características de una persona ordenada, responsable, pulcro y organizado; los de 
extraversión miden la socialización, egocentrismo, diálogo social y soltura; los de agradabilidad miden sensibilidad, empatía, interés y emotividad; los de estabilidad emocional (neuroticismo) miden cambios de humor, estabilidad emocional, estado de bienestar y variabilidad emocional; y, por último, los de apertura miden creatividad, imaginación, fluidez mental y abstracción de ideas (Donnellan et al., 2006).

\section{Limitaciones}

Una de las limitaciones del estudio es referente a la muestra. A diferencia de algunos estudios latinoamericanos, es necesario aplicar el instrumento con muestras con mayor número de participantes. Por último, otra limitación de este estudio es el uso exclusivo de medidas de autoinforme aplicadas en formato virtual. Debido a que no se puede resolver las dudas de los participantes de forma directa e inmediata, lo cual predispone a errores de medición.

\section{Conclusión}

El modelo final obtiene adecuados índices de ajuste y la invarianza ha sido favorable para determina que no existen diferencia entre grupos. Por otro lado, el análisis de la fiabilidad puede verse alterado por la reducción de ítems, sin embargo, para los factores con tres reactivos $(\mathrm{N}$ y O) se incrementó sus coeficientes. No obstante, estos resultados muestran mejores coeficientes de fiabilidad a diferencia de otros inventarios de personalidad breves sobre el BigFive. Este instrumento es de utilidad para uso de investigación o para obtener un rápido análisis de las características de personalidad, más no se recomienda su uso como instrumento de diagnóstico. Su implicancia puede beneficiar el proceso del análisis de la personalidad para investigaciones en el Perú. Por otro lado, se recomienda a la comunidad científica revisar de forma periódica sus propiedades psicométricas.

\section{Referencias}

Anicama, J., \& Chumbimuni, A. (2018). Manual: Cuestionario breve de personalidad de los cinco grandes factores CBP. Fondo Editorial-CIPMOC.

Araujo, E. D. (2014). Cuestionario de personalidad situacional: estudio psicométrico y comparativo en adolescentes de Lima. Revista de Psicología, 16 (2), 139-151. http://revistas.ucv.edu.pe/index.php/R_PSI/article/view/250

Ato, M., López, J. J., \& Benavente, A. (2013). Un sistema de clasificación de los diseños de investigación en psicología. Anales de Psicología, 29 (3), 1038-1059. 
https://doi.org/10.6018/analesps.29.3.178511

Baldasaro, R. E., Shanahan, M. J., \& Bauer, D. J. (2013). Psychometric Properties of the Mini-IPIP in a Large, Nationally Representative Sample of Young Adults. Journal of Personality Assessment, 95 (1), 74-84. https://doi.org/10.1080/00223891.2012.700466

Blanken, T. F., Dekker, K., \& Van Someren, E. J. W. (2018). How personality profile similarity can improve comparability between assessment formats: An example of the Mini-IPIP and IPIP-NEO-120 in a Dutch community sample. PsyArXiv Preprints. https://doi.org/10.31234/osf.io/pjtgv

Browne, M. W., \& Cudeck, R. (1992). Alternative Ways of Assessing Model Fit. Sociological Methods \& Research, 21 (2), 230-258. https://doi.org/10.1177/0049124192021002005

Burisch, M. (1997). Test length and validity revisited. European Journal of Personality, 11 (4), 303-315. https://onlinelibrary.wiley.com/doi/abs/10.1002/\%28SICI\%2910990984\%28199711\%2911\%3A4\%3C303\%3A\%3AAID-PER292\%3E3.0.CO\%3B2-\%23

Campbell, D. T., \& Fiske, D. W. (1959). Convergent and discriminant validation by the multitrait-multimethod matrix. Psychological Bulletin, 56 (2), 81-105. https://doi.org/10.1037/h0046016

Carretta, T. R., Ree, M. J., \& Teachout, M. S. (2012). Measuring Personality in Business: The General Personality Factor in the Mini IPIP. American Journal of Management, 12, 8187. http://na-businesspress.homestead.com/AJM/TeachoutMS_Web12_2_3_.pdf

Cassaretto, M. (2011). Relaciones entre la personalidad y el afrontamiento en estudiantes preuniversitarios. Revista Vanguardia Psicológica Clínica Teórica y Práctica, 1 (2), 202-225. https://dialnet.unirioja.es/servlet/articulo?codigo=4815128

Cattell, R. B. (1943). The description of personality: basic traits resolved into clusters. The Journal of Abnormal and Social Psychology, 38 (4), 476-506. https://doi.org/10.1037/h0054116

Cheung, G. W., \& Rensvold, R. B. (2002). Evaluating Goodness-of-Fit Indexes for Testing Measurement Invariance. Structural Equation Modeling: A Multidisciplinary Journal, 9 (2), 233-255. https://doi.org/10.1207/S15328007SEM0902_5

Choi, J., Fan, W., \& Hancock, G. R. (2009). A Note on Confidence Intervals for Two-Group Latent Mean Effect Size Measures. Multivariate Behavioral Research, 44 (3), 396-406. 
https://doi.org/10.1080/00273170902938902

Cooper, A. J., Smillie, L. D., \& Corr, P. J. (2010). A confirmatory factor analysis of the MiniIPIP five-factor model personality scale. Personality and Individual Differences, 48 (5), 688-691. https://doi.org/10.1016/j.paid.2010.01.004

Costa, P. T., \& McCrae, R. R. (1992). Revised NEO Personality Inventory (NEO PI-R) and NEO Five-Factor Inventory (NEO-FFI). Psychological Assessment Resources.

Czerwiński, S., \& Atroszko, P. (2020). Scores of short and free scale for Big Five explain perceived stress at different stages of life: validity, reliability and measurement invariance of the Polish adaptation of Mini-IPIP. Current Issues in Personality Psychology, 8 (1), 73-82. https://doi.org/10.5114/cipp.2020.95149

Dominguez-Lara, S. A. (2016). Evaluación de la confiabilidad del constructo mediante el coeficiente H: breve revisión conceptual y aplicaciones. Psychologia, 10 (2), 87-94. https://doi.org/10.21500/19002386.2134

Dominguez-Lara, S. A. (2018). Propuesta de puntos de corte para cargas factoriales: una perspectiva de fiabilidad de constructo. Enfermería Clínica, 28 (6), 401-402. https://doi.org/10.1016/j.enfcli.2018.06.002

Dominguez-Lara, S. A., \& Merino-Soto, C. (2018). Dos versiones breves del Big Five Inventory en universitarios peruanos: BFI-15p y BFI-10p. Liberabit. Revista Peruana de Psicología, 24 (1), 81-96. https://doi.org/10.24265/liberabit.2018.v24n1.06

Dominguez-Lara, S. A., Merino-Soto, C., Zamudio, B., \& Guevara-Cordero, C. (2018). Big Five Inventory en Universitarios Peruanos: Resultados Preliminares de su Validación. Psykhe (Santiago), 27 (2), 1-12. https://doi.org/10.7764/psykhe.27.2.1052

Donnellan, M. B., Oswald, F. L., Baird, B. M., \& Lucas, R. E. (2006). The Mini-IPIP scales: Tiny-yet-effective measures of the Big Five factors of personality. Psychological Assessment, 18 (2), 192-203. https://doi.org/10.1037/1040-3590.18.2.192

Eysenck, H. J., \& Eysenck, S. B. G. (1964). Manual of the Eysenck Personality Inventory. London University.

Fiske, D. W. (1949). Consistency of the factorial structures of personality ratings from different sources. Journal of Abnormal and Social Psychology, 44 (3), 329-344. https://doi.org/10.1037/h0057198 
Gastelumendi, C., \& Oré, B. (2013). Personalidad y afrontamiento en voluntarios peruanos de lucha contra la pobreza. Revista de Psicología, 31 (1), 67-98. https://doi.org/10.18800/psico.201301.003

George, D., \& Mallery, P. (2020). IBM SPSS Statistics 26: Step by Step. A Simple Guide and Reference (6ta ed.). New York: Taylor \& Francis.

Goldberg, L. R. (1981). Language and individual differences: The search for universals in personality lexicons. In L. Wheeler (Ed.), Review of Personality and Social Psychology (pp. 141-165). SAGE Publications.

Goldberg, L. R. (1992). The development of markers for the Big-Five factor structure. Psychological Assessment, 4 (1), 26-42. https://doi.org/10.1037/1040-3590.4.1.26

Goldberg, L. R. (1999). A broad-bandwidth, public-domain, personality inventory measuring the lower level facets of several Five-Factor models. En I. Mervielde, I. J. Deary, F. De Fruyt, \& F. Ostendorf (Eds.), Personality psychology in Europe (7ma ed., pp. 7-28). Tilburg University Press.

Gosling, S. D., Rentfrow, P. J., \& Swann, W. B. (2003). A very brief measure of the Big-Five personality domains. Journal of Research in Personality, 37 (6), 504-528. https://doi.org/10.1016/S0092-6566(03)00046-1

Hernández Muro, R. A. (2013). Relación entre las dimensiones de personalidad y la presencia de conductas de agresión en adolescentes varones de un colegio particular de Lima Metropolitana. Revista Psicológica Herediana, 8 (1-2), 32. https://doi.org/10.20453/rph.v8i1-2.2947

John, O. P., Donahue, E. M., \& Kentle, R. L. (1991). The Big Five Inventory versions - 4a and 54. Institute of Personality and Social Research.

Jones, W. P. (2014). Enhancing a Short Measure of Big Five Personality Traits With Bayesian Scaling. Educational and Psychological Measurement, 74 (6), 1049-1066. https://doi.org/10.1177/0013164414525040

Laverdière, O., Gamache, D., Morin, A. J. S., \& Diguer, L. (2020). French adaptation of the Mini-IPIP: A short measure of the Big Five. European Review of Applied Psychology, 70 (3), 100512. https://doi.org/10.1016/j.erap.2019.100512

Laverdière, O., Morin, A. J. S., \& St-Hilaire, F. (2013). Factor structure and measurement 
invariance of a short measure of the Big Five personality traits. Personality and Individual Differences, 55 (7), 739-743. https://doi.org/10.1016/j.paid.2013.06.008

Leong, F. W., Mohd Yasin, M. A., Muhd Ramli, E. R., Fadzil, N. A., \& Kueh, Y. C. (2019). Validation of the Malay Version of Mini-IPIP among Substance Use Disorder Patients Attending Methadone Clinics in Malaysia. International Journal of Environmental Research and Public Health, 16 (22), 4434. https://doi.org/10.3390/ijerph16224434

Li, Z., Sang, Z., Wang, L., \& Shi, Z. (2012). The Mini-IPIP Scale: Psychometric Features and Relations with PTSD Symptoms of Chinese Earthquake Survivors. Psychological Reports, 111 (2), 641-651. https://doi.org/10.2466/16.12.15.PR0.111.5.641-651

Martínez-Molina, A., \& Arias, V. B. (2018). Balanced and positively worded personality short-forms: Mini-IPIP validity and cross-cultural invariance. PeerJ, 6 (9), e5542. https://doi.org/10.7717/peerj.5542

McCrae, R. R., \& Costa, P. T. (1987). Validation of the Five-Factor Model of Personality Across Instruments and Observers. Journal of Personality and Social Psychology, 52 (1), 81-90. https://doi.org/10.1037/0022-3514.52.1.81

Millon, T. (1997). Millon Clinical Multiaxial Inventory III (MCMI III) (2nd ed.). National Computers Systems.

Norman, W. T. (1963). Toward an adequate taxonomy of personality attributes: Replicated factor structure in peer nomination personality ratings. Journal of Abnormal and Social Psychology, 66 (6), 574-583. https://doi.org/10.1037/h0040291

Ock, J., McAbee, S. T., Mulfinger, E., \& Oswald, F. L. (2020). The Practical Effects of Measurement Invariance: Gender Invariance in Two Big Five Personality Measures. Assessment, 27 (4), 657-674. https://doi.org/10.1177/1073191119885018

Oliveira, J. P. (2019). Psychometric Properties of the Portuguese Version of the Mini-IPIP five-Factor Model Personality Scale. Current Psychology, 38 (2), 432-439. https://doi.org/10.1007/s12144-017-9625-5

Perry, L. M., Hoerger, M., Molix, L. A., \& Duberstein, P. R. (2020). A Validation Study of the Mini-IPIP Five-Factor Personality Scale in Adults With Cancer. Journal of Personality Assessment, 102 (2), 153-163. https://doi.org/10.1080/00223891.2019.1644341 
Rhemtulla, M., Brosseau-Liard, P. É., \& Savalei, V. (2012). When can categorical variables be treated as continuous? A comparison of robust continuous and categorical SEM estimation methods under suboptimal conditions. Psychological Methods, 17 (3), 354373. https://doi.org/10.1037/a0029315

Robins, R. W., Hendin, H. M., \& Trzesniewski, K. H. (2001). Measuring Global Self-Esteem: Construct Validation of a Single-Item Measure and the Rosenberg Self-Esteem Scale. Personality and Social Psychology Bulletin, 27 (2), 151-161. https://doi.org/10.1177/0146167201272002

Robins, R. W., Tracy, J. L., Trzesniewski, K., Potter, J., \& Gosling, S. D. (2001). Personality Correlates of Self-Esteem. Journal of Research in Personality, 35 (4), 463-482. https://doi.org/10.1006/jrpe.2001.2324

Rosas, A., Llanos, F., Mendoza, D., Contreras, C., \& Huayta, E. (2001). Perfil de personalidad de estudiantes de la Facultad de Medicina de la Universidad Peruana Cayetano Heredia. Revista de Neuro-Psiquiatria, 64 (1), 51-70. https://doi.org/10.20453/rnp.v64i1.1480

Russell, J. A., Weiss, A., \& Mendelsohn, G. A. (1989). Affect Grid: A single-item scale of pleasure and arousal. Journal of Personality and Social Psychology, 57 (3), 493-502. https://doi.org/10.1037/0022-3514.57.3.493

Sánchez, H. H., \& Reyes, C. (2015). Metodología y diseños en la investigación científica. Visión Universitaria.

Sarria-Joya, C., LLaja, V., García, P., \& Salazar C., M. (2011). Validación del scanning neuropsicológico de la personalidad en estudiantes universitarios. Revista de Investigación en Psicología, 14 (1), 261-272. https://doi.org/10.15381/rinvp.v14i1.2086

Sibley, C. G., Luyten, N., Purnomo, M., Mobberley, A., Wootton, L. W., Hammond, M. D., Sengupta, N., Perry, R., West-Newman, T., Wilson, M. S., McLellan, L., Hoverd, W. J., \& Robertson, A. (2011). The Mini-IPIP6: Validation and extension of a short measure of the Big-Six factors of personality in New Zealand. New Zealand Journal of Psychology, 40 (3), 142-159.

Simkin, H., Borchardt-Dutera, L., \& Azzollini, S. (2020). Evidencias de validez del Compendio Internacional de Ítems de Personalidad Abreviado. Liberabit: Revista Peruana de Psicología, 26 (1), 1-23. https://doi.org/10.24265/liberabit.2020.v26n1.02 
Topolewska, E., Skimina, E., Strus, W., Cieciuch, J., \& Rowiński, T. (2014). The Short IPIPBFM-20 Questionnaire for Measuring The Big Five. Roczniki Psychologiczne, 17 (2), 385-402. http://czasopisma.tnkul.pl/index.php/rpsych/article/view/3165

Vizcarra Fernández, M. Á., Llaja Rojas, V., Limo Sánchez, C., \& Talavera Aguilar, J. (2015). Clima laboral, Burnout y Perfil de Personalidad: Un estudio en personal asistencial de un Hospital Público de Lima. Informes Psicológicos, 15 (2), 111-126. https://doi.org/10.18566/nfpsicv15n2a06

Wielkiewicz, R. M. (2015). Confirmatory and exploratory factor analysis of the Mini-IPIP with a multi-institutional sample of first- year college students. Psychology Faculty Publication, 5, 1-17. http://digitalcommons.csbsju.edu/psychology_pubs/93

Wise, S. L., \& DeMars, C. E. (2005). Low Examinee Effort in Low-Stakes Assessment: Problems and Potential Solutions. Educational Assessment, 10 (1), 1-17. https://doi.org/10.1207/s15326977ea1001_1

Yuan, K.-H., \& Bentler, P. M. (1998). Structural Equation Modeling with Robust Covariances. Sociological Methodology, 28 (1), 363-396. https://doi.org/10.1111/00811750.00052 